17. Kleist $P(2001)$ Immer noch Waisenkinder in der Medizin. Schweiz Ärztez 82:2221-2229

18. Mergel A, Mentzer D, Keller-Stanislawski B (2012) UAW-Meldung durch den Patienten - spezielles Internetportal geplant. Pharmakovigilanz Transparent 2012:18-22

19. Neubert A, Rascher W (2007) Unerwünschte Arzneimittelwirkungen bei Kindern. Monatsschr Kinderheilkd 155:700-708

20. Neubert A, Dormann H, Weiss J et al (2004) The impact of unlicensed and off-label drug use on adverse drug reactions in paediatric patients. Drug Saf 27:1059-1067

21. Neubert A, Dormann H, Weiss J et al (2006) Are computerised monitoring systems of value to improve pharmacovigilance in paediatric patients? Eur J Clin Pharmacol 62:959-965

22. Oehme AK, Rashed AN, Hefele B et al (2012) Adverse drug reactions in hospitalised children in Germany are decreasing: results of a nine year cohort-based comparison. PLoS One 7:e44349

23. Pandolfini C, Bonati M (2005) A literature review on off-label drug use in children. Eur J Pediatr 164:552-558

24. Paul-Ehrlich-Institut (2012) Meldung von Verdachtsfällen unerwünschter Arzneimittelwirkungen und Impfkomplikationen. PEI, Berlin. http:// humanweb.pei.de/. Zugegriffen: 21.12.2012

25. Rascher W, Mentzer D, Seyberth HW (2007) Verbesserung der Arzneimittelsicherheit durch Pharmakovigilanz. Monatsschr Kinderheilkd 155:692-699

26. Rashed AN, Neubert A, Tomlin S et al (2012) Epidemiology and potential associated risk factors of drug-related problems in hospitalised children in the United Kingdom and Saudi Arabia. Eur J Clin Pharmacol 68:1657-1666

27. Rashed AN, Wong IC, Cranswick N et al (2012) Adverse drug reactions in children - international surveillance and evaluation (ADVISE): a multicentre cohort study. Drug Saf 35:481-494

28. Sanz EJ, De-las-Cuevas C, Kiuru A et al (2005) Selective serotonin reuptake inhibitors in pregnant women and neonatal withdrawal syndrome: a database analysis. Lancet 365:482-487

29. Standing JF, Tuleu C (2005) Paediatric formulations - getting to the heart of the problem. Int J Pharm 300:56-66

30. Star K, Noren GN, Nordin K et al (2011) Suspected adverse drug reactions reported for children worldwide: an exploratory study using VigiBase. Drug Saf 34:415-428

31. The ADDUCE Consortium (2011) ADDUCE - attention deficit hyperactivity disorder drugs use chronic effects. ADDUCE, Dundee. http://adhd-adduce. org. Zugegriffen: 01.08.2011

32. Verhamme K, Bonifazi F, Ceci A et al (2009) Adverse drug reactions reporting in children. Pharmaceut Policy Law 2009:89-99

33. Wong IC, Basra N, Yeung VW et al (2006) Supply problems of unlicensed and off-label medicines after discharge. Arch Dis Child 91:686-688
Monatsschr Kinderheilkd 2013 · 161:308-315 DOI 10.1007/s00112-013-2899-2

(c) Springer-Verlag Berlin Heidelberg 2013

\section{F. Wild}

Wissenschaftliches Institut der Privaten Krankenversicherung (WIP), Köln

\section{Erratum zu: Impfpraxis gegen humane Papillomaviren für 2006-2011 - Evaluation anhand von Arznei- verordnungsdaten der privaten Krankenversicherung}

\section{Monatsschr Kinderheilkd 161:34-39 http://dx.doi.org/10.1007/s00112-012-2786-2}

Einige Angaben zum im Text erwähnten HPV-Impfstoff (HPV: humanes Papillomavirus) Gardasil ${ }^{\oplus}$ der Firma Sanofi Pasteur MSD GmbH entsprechen nicht den aktuellen Fachinformationen. Gardasil ${ }^{\circ}$ ist zur Anwendung bei beiden Geschlechtern ab einem Alter von 9 Jahren zugelassen. Eine Altersbeschränkung nach oben, wie im Text beschrieben, existiert nicht. Gardasil $^{\circledR}$ schützt laut der aktuellen Fachinformation zudem vor Erkrankungen, die durch die HPV-Typen 6, 11, 16 und 18 (und nicht wie im Text erwähnt 6, 11, 17 und 19) verursacht werden.

\section{Korrespondenzadresse}

\section{Dr. F. Wild}

Wissenschaftliches Institut der Privaten Krankenversicherung (WIP)

Gustav-Heinemann-Ufer 74c, 50968 Köln

Frank.Wild@pkv.de 